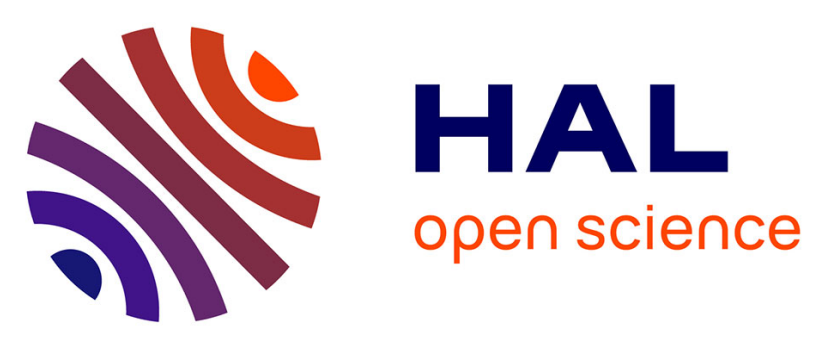

\title{
Des flux d'eau aux flux de matières en suspension et de contaminants associés: gestion d'un réseau de stations hydro-sédimentaires sur le Rhône
}

C. Le Bescond, Fabien Thollet, G. Poulier, S. Gairoard, H. Lepage, F. Branger, L. Jamet, N. Raidelet, O. Radakovitch, Aymeric Dabrin, et al.

\section{To cite this version:}

C. Le Bescond, Fabien Thollet, G. Poulier, S. Gairoard, H. Lepage, et al.. Des flux d'eau aux flux de matières en suspension et de contaminants associés: gestion d'un réseau de stations hydrosédimentaires sur le Rhône. La Houille Blanche - Revue internationale de l'eau, 2018, 3, pp.63-70. 10.1051/lhb/2018033 . hal-01945337

\section{HAL Id: hal-01945337 \\ https://hal.science/hal-01945337}

Submitted on 5 Dec 2018

HAL is a multi-disciplinary open access archive for the deposit and dissemination of scientific research documents, whether they are published or not. The documents may come from teaching and research institutions in France or abroad, or from public or private research centers.
L'archive ouverte pluridisciplinaire HAL, est destinée au dépôt et à la diffusion de documents scientifiques de niveau recherche, publiés ou non, émanant des établissements d'enseignement et de recherche français ou étrangers, des laboratoires publics ou privés. 


\title{
Des flux d'eau aux flux de matières en suspension et de contaminants associés : gestion d'un réseau de stations hydro-sédimentaires sur le Rhône
}

\author{
Chloé LE BESCOND ${ }^{1,2}$, Fabien THOLLET ${ }^{1}$, Gaëlle POULIER ${ }^{2}$, Stéphanie GAIROARD ${ }^{3}$, Hugo LEPAGE4, \\ Flora BRANGER ${ }^{1}$, Luc JAMET ${ }^{5}$, Nicolas RAIDELET ${ }^{5}$, Olivier RADAKOVITCH ${ }^{3}$, Aymeric DABRIN ${ }^{2}$, \\ Marina COQUERY ${ }^{2}$, Jérôme LE COZ ${ }^{1}$
}

\author{
Irstea, UR HHLY, centre de Lyon-Villeurbanne, 5 rue de la Doua, BP 32108, F-69616 Villeurbanne Cedex, France chloe.le-bescond@irstea.fr \\ 2. Irstea, UR MALY, centre de Lyon-Villeurbanne, F-69616 Villeurbanne, France. \\ 3. Cerege, Aix Marseille Université, INSU, IRD, CNRS : UMR7330, Europôle Méditerranéen de l'Arbois, 13545 Aix-en-Provence, France. \\ 4. IRSN, LRTE, 13115 Saint Paul Lez Durance, France. \\ 5. Irstea, DSI, pôle Informatique Scientifique, centre de Lyon-Villeurbanne, F-69626 Villeurbanne, France.
}

RÉSUMÉ. - La Base de Données pour les Observatoires en Hydrologie (BDOH) est un outil de bancarisation qui permet de combiner les mesures de débit, de matières en suspension (MES) et de contaminants particulaires issues d'un même réseau de stations pour calculer des chroniques de flux instantanés et cumulés. Cette application est utilisée dans le cadre de l'Observatoire des Sédiments du Rhône (OSR) pour estimer les flux de MES et de contaminants associés (Hg, PCB,

...) en transit dans le Rhône et ses principaux affluents. Cependant, avant le calcul de ces chroniques, les données mères lacunaires doivent être reconstituées. Un test de sensibilité a été mené afin d'évaluer l'impact de différentes méthodes d'estimation des chroniques de concentrations en MES et en Hg particulaire sur les calculs de flux instantanés et cumulés dans le Rhône aux deux stations principales de l'OSR (Jons et Arles). Les résultats montrent que, quelle que soit la méthode d'estimation utilisée, les flux annuels de MES et de $\mathrm{Hg}$ particulaire ne diffèrent pas plus de $5 \%$. A l'échelle mensuelle, en revanche, les différentes méthodes génèrent des variabilités plus élevées (26-51\%). En perspective, ces premiers résultats seront complétés par l'évaluation des incertitudes quantitatives sur les calculs de flux particulaires.

Mots-clés : Base de données, débit, flux particulaire, sédiments, série temporelle

\section{From water fluxes to suspended particulate matter and associated contaminant fluxes: management of hydro-sedimentary stations on the Rhône River}

\begin{abstract}
The database for hydrological observatories (BDOH) is an efficient tool for storage which allows the combination of water discharge data, suspended particulate matter (SPM) and particulate contaminants concentrations, produced thought a same observatory network, to calculate time series fluxes. This application is used in the Rhône Sediment Observatory (OSR) program to estimate SPM and their associated contaminants fluxes along the Rhône River and its main tributaries. However, incomplete time series need to be filled prior to computation in BDOH. Hence, a sensitivity test was run to evaluate the effect of different estimation methods of SPM concentration and particulate mercury time series on final instantaneous and accumulated fluxes on the Rhône River at the two main stations of the OSR network (Jons and Arles). The results demonstrate that the differences between SPM and particulate mercury annual fluxes assessed with different estimation methods are less than $5 \%$. However, at monthly scale, estimation methods can create higher differences $(26-51 \%)$. In the future, these preliminary results will be completed with the estimation of the uncertainties associated with particle flux measurements.
\end{abstract}

Key words: Database, water discharge, particulate flux, sediment, time series

\section{INTRODUCTION}

Le Rhône représente la principale source de matières en suspension (MES) à la mer Méditerranée [Ferrand et al., 2012]. En 2009, l'Observatoire des Sédiments du Rhône (OSR) a été créé pour acquérir des données visant à quantifier de manière robuste les flux de MES et de contaminants associés (mercure $[\mathrm{Hg}]$, métaux, polychlorobiphényles [PCB], et radionucléides) en transit dans le Rhône, du lac Léman à la mer Méditerranée, à des échelles temporelles allant d'une crue journalière à des bilans annuels. Pour répondre à cet objectif, des stations hydrométriques préexistantes gérées par l'OFEV, la CNR et la DREAL' ont été instrumentées pour assurer le suivi en continu des concentrations en MES et en contaminants particulaires. Ce réseau d'observation multi-partenarial comprend deux stations principales sur le Rhône à Jons et à Arles, complétées par des stations secondaires installées sur les affluents majeurs (e.g. Arve, Saône, Isère, Durance) pour identifier la

1. Office fédéral Suisse de l'environnement (OFEV), Compagnie Nationale du Rhône (CNR), Direction Régionale de l'Environnement, de l'Aménagement et du Logement (DREAL) 
contribution de chaque sous-bassin-versant en termes de flux de MES et de contaminants associés (Cf. Figure 1).

Les séries temporelles brutes générées à travers ce réseau d'observation, appelées chroniques mères, sont mises à disposition sur Internet ${ }^{2}$. Cette Base de Données des Observatoires en Hydrologie (BDOH) a été développée par Irstea pour bancariser les données issues d'observatoires hydrologiques de long terme [Branger et al., 2014]. A partir de séries temporelles mères, $\mathrm{BDOH}$ permet de calculer automatiquement des chroniques de flux instantanés et cumulés de MES et de contaminants particulaires. Cependant, les chroniques acquises sur le terrain contiennent inévitablement des lacunes, qui doivent être reconstituées avant le lancement des calculs de flux cumulés dans BDOH. L'objectif de cette étude est d'évaluer la sensibilité des méthodes d'estimation des chroniques mères sur les calculs de flux instantanés et cumulés. Le test s'est focalisé sur les chroniques mères de concentrations en MES et en mercure associé aux deux stations principales de l'OSR. Les données manquantes ont été comblées selon trois techniques. Les résultats des flux calculés à partir de ces chroniques reconstituées ont ensuite été comparés pour permettre de définir une stratégie d'estimation des chroniques selon les paramètres étudiés.

\section{STRATÉGIE D'OBSERVATION DES FLUX DE MES ET DE CONTAMINANTS ASSOCIÉS}

\section{II.1. Les séries temporelles mères}

Les séries de débits et de concentrations en MES sont enregistrées en continu à un pas de temps fin (de 10 minutes à une heure) sur toutes les stations du réseau d'observation. Les concentrations en contaminants sont suivies toutes les deux semaines aux stations principales et tous les mois aux stations permanentes et temporaires. La stratégie d'observation des flux décrite ci-dessous se focalise sur les deux stations principales à Jons et à Arles.

2. https://bdoh.irstea.fr/OBSERVATOIRE-DES-SEDIMENTS-DU-RHONE/

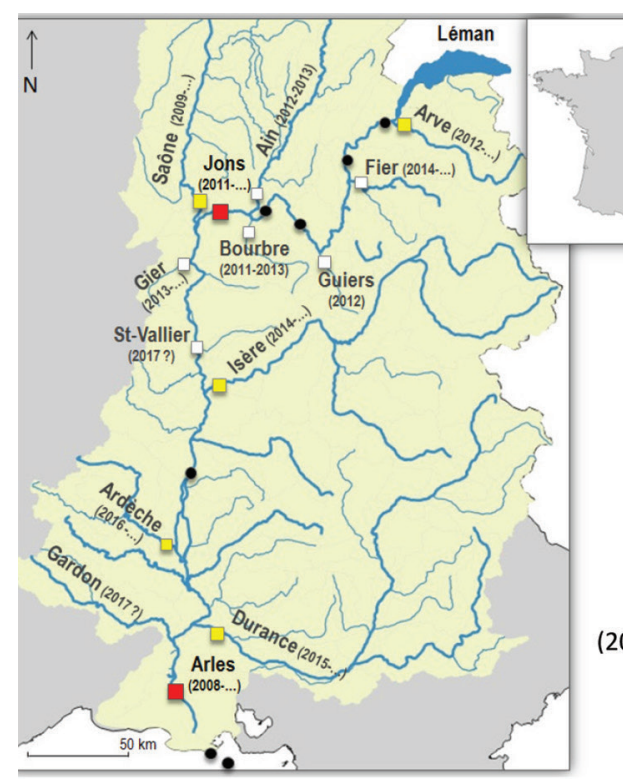

\section{II.1.1. Le suivi des débits}

Le débit moyen horaire du Rhône à Jons (en $\left.\mathrm{m}^{3} / \mathrm{s}\right)$ est simulé à l'aide du modèle hydraulique Rhône1D [Dugué et al., 2015] à partir des hydrogrammes du Rhône à Lagnieu (données CNR), de l'Ain à Port-Galland (données CNR) et de la Bourbre à Tignieu-Jameyzieu (données DREAL Rhône-Alpes).

Le débit mesuré sur le Grand Rhône à Arles est fourni par la CNR au pas de temps horaire.

\section{II.1.2. Le suivi des concentrations en matières en suspension}

La station de Jons est équipée d'un capteur de turbidité (en NTU), géré par le Grand Lyon, qui mesure la réduction de la transparence de l'eau due à la présence de matières en suspension [AFNOR, 2016]. Les données sont enregistrées en continu à un pas de temps de 10 minutes puis validées à l'aide du logiciel DP+ développé par la DREAL. Cette étape consiste à vérifier la cohérence des données en les comparant à celles d'un paramètre lié (le débit et la turbidité par exemple), à supprimer manuellement des valeurs aberrantes, à recaler les valeurs en cas de dérive du capteur et à identifier les périodes de lacune. En parallèle, un échantillonneur automatique ISCO 3700 prélève des échantillons d'eau horodatés pour la détermination des concentrations en MES par filtration et pesée [AFNOR, 2005]. Ces résultats permettent de construire la courbe de calibration turbidité/MES [Navratil et al., 2011] du capteur avec le logiciel BaRatin [Le Coz et al., 2014] (Cf. Figure 2). L'équation de la droite de régression, forcée par 0, est stockée dans $\mathrm{BDOH}$ pour transformer la chronique de turbidité en chronique de concentration en MES (en mg/L). Ce facteur de conversion est utilisable pour une période d'application et une plage de validité définies et peut être complété lorsque la relation turbidité/MES du capteur est modifiée. La série temporelle calculée de MES est vérifiée tous les mois grâce à des analyses ponctuelles de MES.

A la station d'Arles, les concentrations en MES sont mesurées par plusieurs partenaires (MIO/IRSN/Cerege) sur des prélèvements d'eau composites réalisés quotidiennement [AFNOR, 2005].

Figure 1 : Les stations de mesures de l'Observatoire des Sédiments du Rhône au 01/09/2016. 


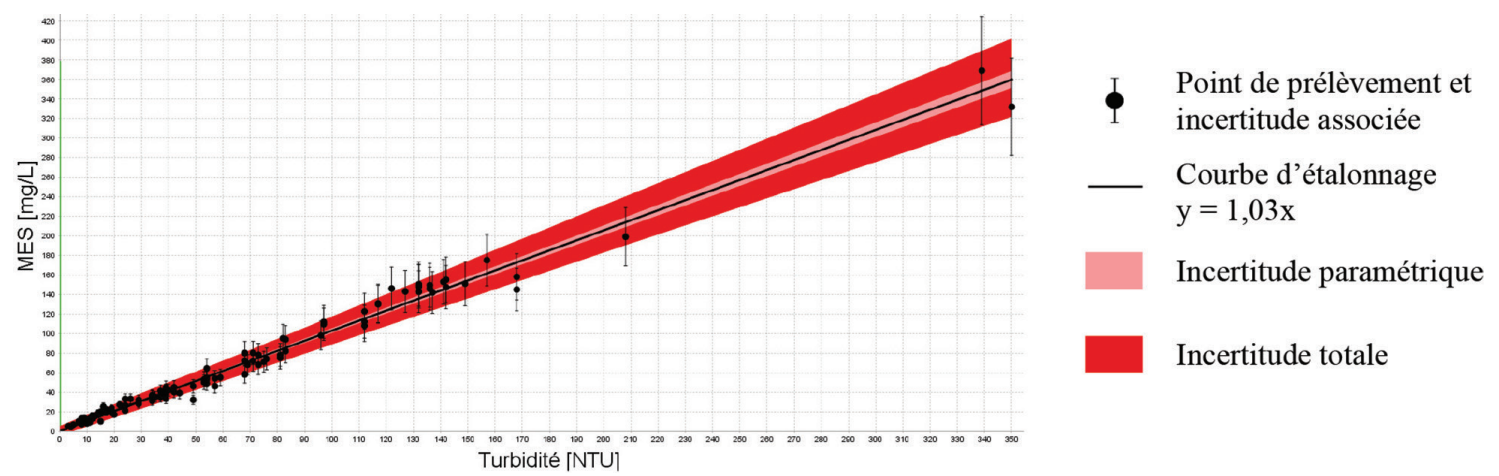

Figure 2 : Courbe de calibration du turbidimètre de Jons au 01/10/16, établie à l'aide du logiciel BaRatin.

\section{II.1.3. Le suivi des concentrations en contaminants particulaires}

Le suivi des concentrations en contaminants s'effectue sur des échantillons de MES collectés à l'aide d'une centrifugeuse en continu (CEPA Z61). Les prélèvements sont réalisés toutes les deux semaines en régime de base et plus fréquemment en cas de crue, sur une durée généralement comprise entre 30 minutes et 4 heures. Les échantillons de centrifugation sont considérés comme représentatifs des MES qui transitent dans le Rhône [Launay, 2014]. Parallèlement à ces prélèvements " ponctuels », des prélèvements intégratifs de MES sont réalisés à Jons grâce à un piège à particules [Schulze et al., 2007], pour permettre d'échantillonner les pics de flux de MES sur une longue période. Ce dispositif, nécessitant une maintenance aisée et peu coûteuse, se présente sous la forme d'une boîte en inox $316(40 \times 30 \times 25 \mathrm{~cm})$ percée de 3 trous sur les faces avant et arrière pour faire circuler l'eau. A l'intérieur, 2 cloisons font chuter la vitesse de l'eau, entraînant la décantation des MES au fond de 2 paniers amovibles. La représentativité des échantillons issus du piège est validée par comparaison avec la chronique de contaminant obtenue par centrifugation, considérée comme une référence ponctuelle. Quel que soit le mode de prélèvement utilisé, les chroniques de contaminants sont discontinues, c'est-à-dire qu'il n'y a pas d'interpolation linéaire entre deux concentrations successives, mais une valeur constante sur toute la durée du prélèvement.

Les séries temporelles de débit, de concentrations en MES et en contaminants sont bancarisées dans BDOH [Thollet et al., 2015] avec les dates et heures exactes de début et de fin de prélèvement (Cf. Figure 3). Chaque donnée est nécessairement accompagnée d'un indice de qualité attribué par son producteur, comme par exemple le code « LQ \% signifiant que la concentration est inférieure à la limite de quantification (LQ) de la méthode, ou le code « $\mathrm{E} \%$ pour l'identification d'une donnée reconstituée.

\section{II.2. La méthode d'estimation des flux dans BDOH}

Dans l'hypothèse où les concentrations en MES et en contaminants particulaires sont homogènes au sein de la section du cours d'eau, le flux instantané d'un contaminant particulaire est défini comme le produit du débit, de la concentration en MES et de la concentration du contaminant particulaire considéré [Launay, 2014]. Les chroniques de flux instantanés sont calculées de manière automatique dans $\mathrm{BDOH}$ en multipliant les séries mères entre elles.

Une chronique instantanée de flux de MES est obtenue en multipliant la chronique mère de débit d'une station donnée par la chronique de concentration en MES correspondante. Les deux chroniques mères sont mises au même pas de temps (variable) par interpolation linéaire à la seconde près entre deux points pour ne pas perdre d'information.

Pour calculer une chronique instantanée de flux de contaminant, la chronique discontinue de concentration en contaminant est dans un premier temps transformée en chronique continue, en supposant que les concentrations varient linéairement entre deux valeurs successives. Chaque valeur est reliée à la suivante par un palier centré sur le point de prélèvement (Cf. Figure 4), à l'aide d'un script développé avec

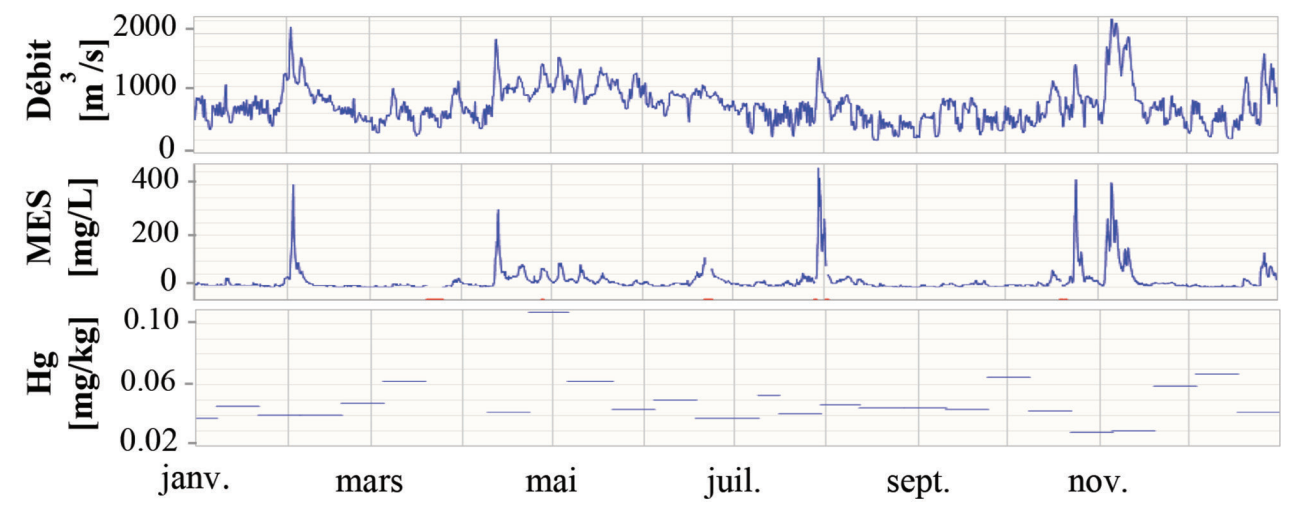

Figure 3 : Séries temporelles de débit $\left(\mathrm{m}^{3} / \mathrm{s}\right)$, de concentration en MES $(\mathrm{mg} / \mathrm{L})$ et de concentration en $\mathrm{Hg}(\mathrm{mg} / \mathrm{kg})$ sur le Rhône à Jons en 2013 visualisées dans BDOH (en bleu les données mesurées, en rouge les lacunes dans la chronique continue de MES). 

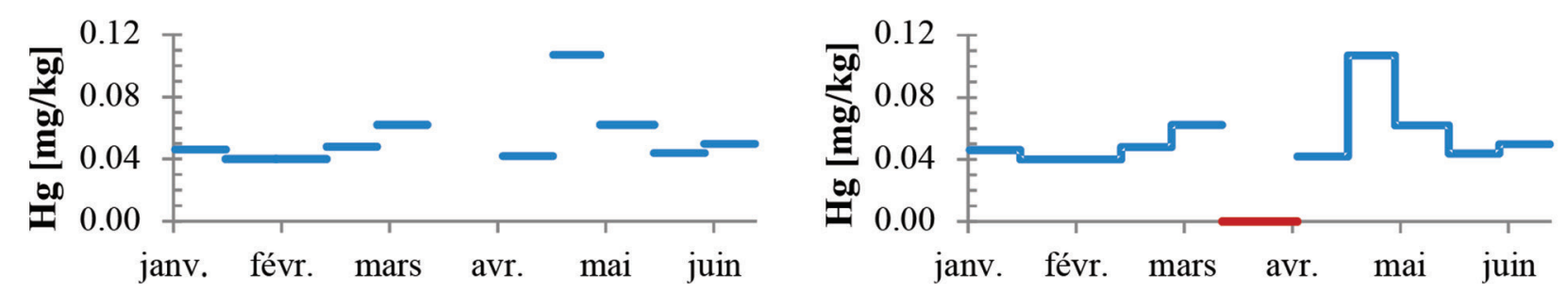

Figure 4 : Transformation de la série temporelle intégrative de Hg discontinue (à gauche) en série continue (à droite) sur la station du Rhône à Jons de janvier à juin 2013. En bleu les données mesurées, en rouge la période de lacune.

le logiciel R. Une période de lacune est identifiée dans la chronique lorsque la fréquence de suivi régulier n'a pas été respectée, par omission d'un prélèvement (panne de la centrifugeuse, piège renversé) ou d'une analyse (tous les échantillons prélevés ne sont pas systématiquement analysés). Ainsi, les séries temporelles des concentrations en contaminants issues de centrifugation sont considérées lacunaires au-delà de 14 jours entre deux données. Les séries temporelles de concentrations en contaminants à Jons issues du piège à particules sont jugées incomplètes dès lors que la date de mise à l'eau d'un piège diffère de la date de collecte de l'échantillon précédent.

Puis, la chronique continue de concentration en contaminant est multipliée par la chronique de flux instantané de MES précédemment calculée pour obtenir la chronique de flux de contaminant particulaire instantané à cette station.

L'indice de qualité affecté à chaque donnée d'une chronique fille correspond au plus défavorable des deux données mères multipliées. En cas d'une donnée non quantifiée dans une chronique mère, la chronique de flux peut être calculée en remplaçant la donnée au choix par i) la valeur de la $L Q$, ii) la moitié de la valeur de la LQ ou iii) une valeur constante qui peut être égale à 0 par exemple.

Cependant, les séries temporelles acquises en continu sur les stations d'observation sont généralement incomplètes. L'absence de données peut provenir de données invalidées car jugées aberrantes lors de la validation ou de lacunes issues, par exemple, par une panne de capteur ou du système de prélèvement. Ces lacunes sont propagées dans les séries de flux instantanés dérivées et empêchent les calculs de flux cumulés sur les périodes concernées. Il est donc nécessaire de reconstituer au préalable les périodes de lacunes dans les chroniques mères continues. Un test de sensibilité aux méthodes d'estimation des chroniques de concentrations en MES et en $\mathrm{Hg}$ particulaire sur les calculs de flux instantanés et cumulés a été mené. L'étude a été réalisée aux stations principales de Jons et d'Arles pour l'année civile 2013. Les résultats de flux ont été comparés pour différentes échelles temporelles.

\section{TEST DE SENSIBILITÉ AUX MÉTHODES DE RECONSTITUTION DES LACUNES}

\section{III.1. Les méthodes de reconstitution testées}

Les séries de débit fournies par la CNR contiennent 2 heures de lacune par an, observées lors du passage à l'heure d'hiver. Les chroniques du Rhône à Arles et en entrée du modèle Rhône 1D ont été comblées par interpolation linéaire. Le débit simulé du Rhône à Jons ne contient pas de lacune.

Les séries temporelles de concentration en MES sont considérées lacunaires si la période d'absence de données dépasse une heure à Jons et 24 heures à Arles, avec une variation supérieure à $10 \mathrm{mg} / \mathrm{L}$ entre les valeurs encadrant cette période ; si la variation est inférieure à ce seuil, la valeur précédant la lacune est prolongée sur toute la durée de la lacune. La chronique lacunaire de MES à Jons a été estimée i) par interpolation linéaire entre les deux valeurs encadrant la lacune et ii) à l'aide d'une relation entre le débit et la concentration en MES [Horowitz, 2008]. La série d'Arles a été reconstituée i) à l'aide de la relation débit/MES et ii) avec une relation débit/MES pour les faibles débits, complétée par les résultats de la simulation du modèle numérique Rhône 1D pour les forts débits.

Les lacunes dans les chroniques continues de concentration en $\mathrm{Hg}$ issues de centrifugation et du piège à particules ont été comblées i) par interpolation linéaire, ii) en dupliquant la valeur précédant la lacune sur toute la durée de la lacune et iii) avec une concentration moyenne en $\mathrm{Hg}$ particulaire déterminée par gamme de débit (Cf. Tableau 1). Les séries de concentration en $\mathrm{Hg}$ en 2013 sont toutes constituées de données supérieures à la LQ $(0,010 \mathrm{mg} / \mathrm{kg})$. L'influence du choix de la valeur de remplacement des données non quantifiées (LQ, LQ/2 ou constante) sur des calculs de flux n'a donc pas été testée ici.

Les pourcentages annuels et mensuels de données à reconstituer sont présentés dans le Tableau 2.

Tableau 1: Concentrations moyennes en Hg particulaire (en $\mathrm{mg} / \mathrm{kg}$ ) par gamme de débit aux stations de Jons et d'Arles, calculées à partir des données de l'OSR sur la période 2011-2015 (土 écart-type ; nombre de données entre parenthèses).

\begin{tabular}{|l|l|c|c|}
\cline { 3 - 4 } \multicolumn{2}{l|}{} & \multicolumn{1}{l|}{ Le Rhône à Jons } & Le Rhône à Arles \\
\hline \multirow{2}{*}{ Seuil de crue $\left(\mathrm{en} \mathrm{m}^{3} / \mathrm{s}\right)$} & 800 & 3000 \\
\hline \multirow{2}{*}{ Concentration moyenne en Hg particulaire (en mg/kg) } & Base & $0.061 \pm 0.003(47)$ & $0.117 \pm 0.051(92)$ \\
\cline { 2 - 4 } & Crue & $0.048 \pm 0.016(44)$ & $0.087 \pm 0.026(24)$ \\
\cline { 2 - 4 } & Chasse & $0.037 \pm 0.010(4)$ & $/$ \\
\hline
\end{tabular}


Tableau 2 : Pourcentages annuels et mensuels de lacunes dans les chroniques de concentration en MES et en Hg mesurées sur le Rhône aux stations de Jons et d'Arles en 2013.

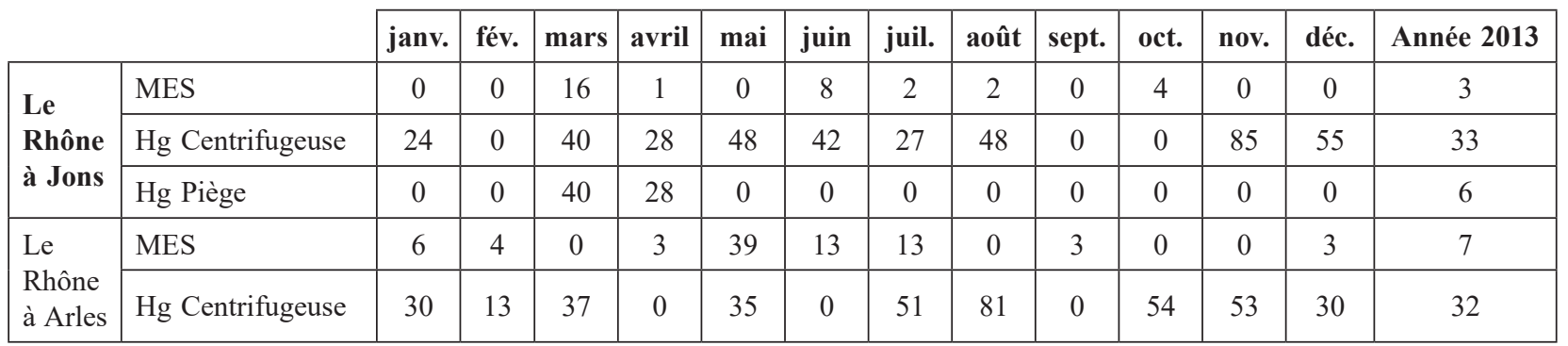

III.2. Les résultats des flux calculés à partir des chroniques reconstituées

\section{III.2.1. Les flux de matières en suspension}

A l'échelle annuelle, les variations entre les cumuls de MES (en Mt) obtenus avec les différentes méthodes de reconstitution testées sont de $1,7 \%$ à Jons et de $0,9 \%$ à Arles. Sur les flux mensuels, les différences s'étendent de
$0 \%$ à $22,5 \%$ (en juin) avec une moyenne de 3,3\% pour Jons, et de $0 \%$ à $1,8 \%$ (en janvier) à Arles, avec une moyenne de $0,6 \%$. D'après ces résultats, les flux annuels et mensuels de MES sont similaires pour les deux méthodes d'estimation testées (Cf. Figure 5).

A l'échelle hebdomadaire, les flux de MES à Jons ont été calculés uniquement sur les semaines comportant des lacunes (Cf. Figure 6). Les différences entre les méthodes

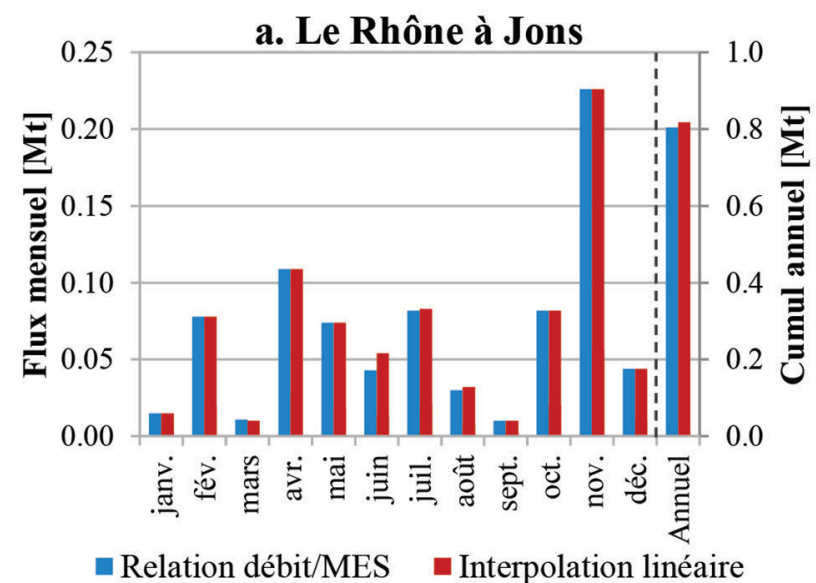

Figure 5 : Flux mensuels et cumuls annuels de MES (Mt) calculés à partir des deux chroniques de MES reconstituées pour l'année 2013 : a) le Rhône à Jons, b) Le Rhône à Arles. Sur chaque graphe, à gauche, l'axe des flux mensuels, à droite l'axe du cumul sur l'année 2013.

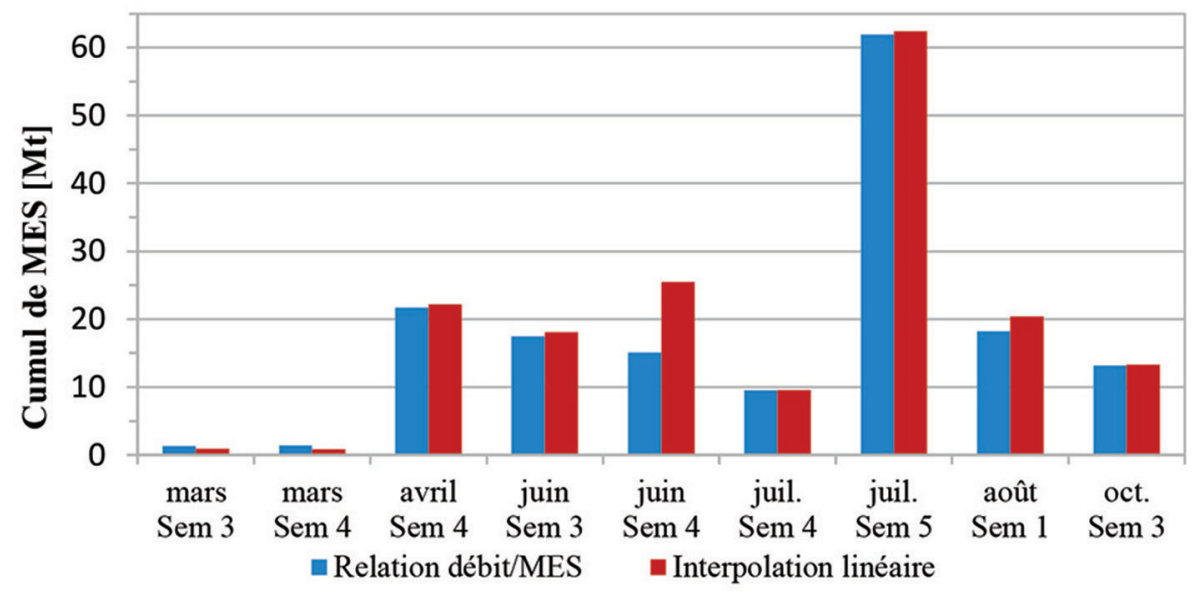

Figure 6 : Flux hebdomadaires de MES (Mt) à Jons calculés à partir des deux chroniques de MES reconstituées sur les semaines lacunaires de l'année 2013. 
de reconstitution sont en général inférieures à $12 \%$. La plus forte variation s'élève à $51 \%$, observée lors de la $4^{\text {ème }}$ semaine de juin, qui pourrait s'expliquer par la variabilité temporelle des concentrations en MES, la période de lacune coïncidant avec un pic de turbidité d'environ 100 NTU. Sur les deux méthodes de reconstitution testées, la relation débit/MES est jugée meilleure car elle prend en compte les variations du débit sur la période de lacune. En juin 2013, cependant, elle sous-estime les concentrations en MES reconstituées à Jons. D'après ces résultats, le calcul de flux cumulé de MES sur une période inférieure à un mois est possible si la chronique de flux de MES instantanée est complète ; en revanche, il est déconseillé lorsque la série contient des lacunes (Cf. III.1).

\section{III.2.2. Les flux de contaminants}

Le croisement des multiples chroniques de MES et de $\mathrm{Hg}$ reconstituées conduit à 12 chroniques de flux de mercure différentes à Jons et à 6 chroniques à Arles. A l'échelle annuelle, les variations des cumuls de $\mathrm{Hg}$ à Jons sont de 4,7\%, tous modes de prélèvements confondus (Cf. Figure 7). En distinguant les cumuls obtenus à partir des deux techniques de prélèvements, les variations sont de $1,6 \%$ entre les 6 chroniques de flux de $\mathrm{Hg}$ issues de centrifugation et de $0,9 \%$ entre celles issues du piège à particules. Les cumuls annuels obtenus par centrifugation sont en moyenne un peu plus élevés que ceux issus du piège : $38,3 \mathrm{~kg}$ pour les centrifugations contre $35,1 \mathrm{~kg}$ pour le piège à particules, soit un écart de $8,4 \%$. Cette différence pourrait s'expliquer par un taux de lacunes plus important dans la chronique de $\mathrm{Hg}$ issue de centrifugation $(33 \%$ sur 2013 et 9 mois comportant des lacunes) que dans celle de mercure issue du piège à particules $(6 \%$ sur 2013 et seulement 2 mois comportant des lacunes). A Arles, les cumuls annuels de mercure sont de $2,8 \%$, malgré un pourcentage élevé de lacunes dans la chronique de mercure (32\% et 9 mois comportant des lacunes). D'après ces tests, les cumuls annuels de $\mathrm{Hg}$ à une station sont similaires quelle que soit la méthode d'estimation.

A l'échelle mensuelle, les résultats sont plus contrastés entre les deux stations (Cf. Figure 8). A Jons, des variations importantes sont parfois observées entre les flux, jusqu'à $26 \%$ de différence en décembre 2013 avec la chronique de mercure issue de centrifugation. Pour un même mois, ces différences varient selon la méthode de prélèvement

\section{a. Le Rhône à Jons}

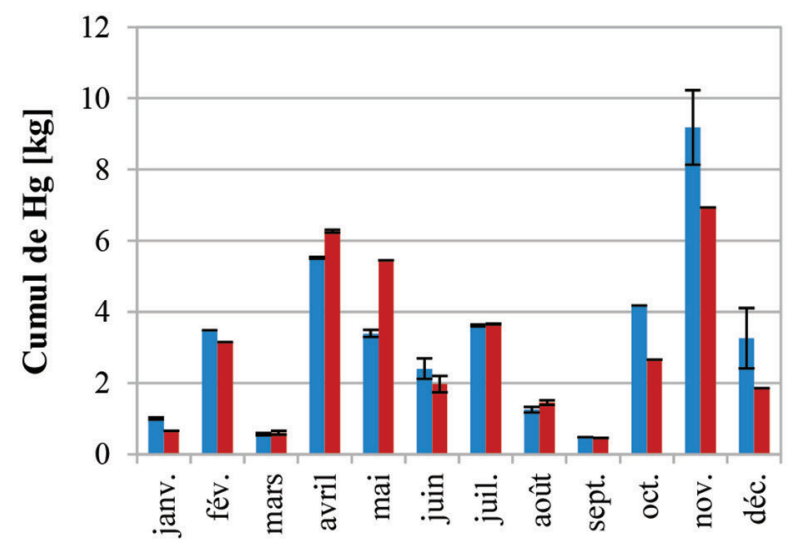

\section{b. Le Rhône à Arles}

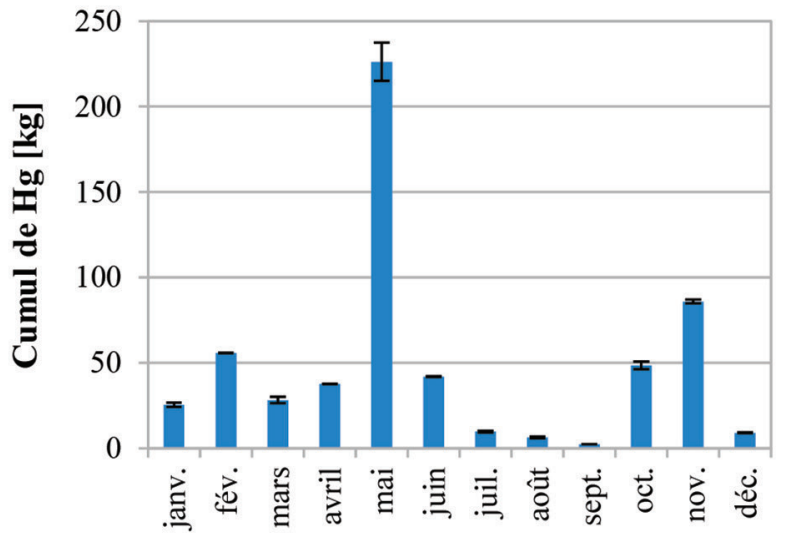

Figure 8 : Flux mensuels de Hg particulaire $(\mathrm{kg})$ en 2013 obtenus avec plusieurs méthodes de reconstitution des lacunes aux stations principales de l'OSR : a) le Rhône à Jons $(n=12)$, b) le Rhône à Arles $(n=6)$. En bleu les données issues de centrifugation, en rouge les données provenant du piège à particules, en noir les écarts-types.
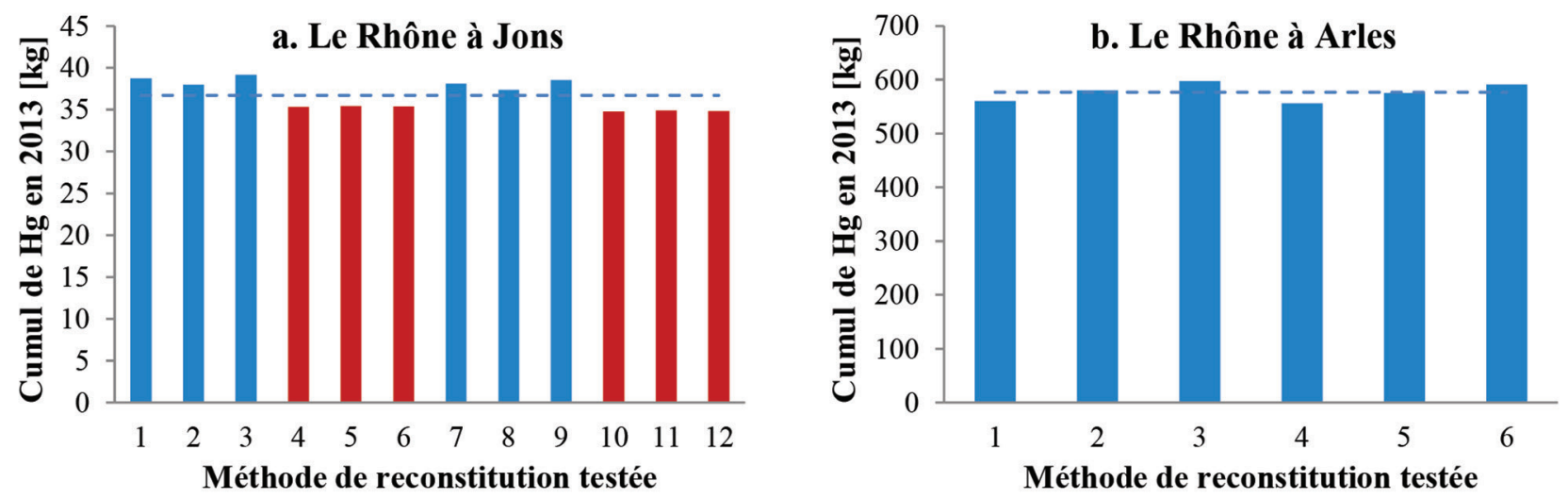

Figure 7 : Cumuls annuels de Hg particulaire $(\mathrm{kg})$ en 2013 obtenus avec plusieurs méthodes de reconstitution des lacunes aux stations principales de l'OSR : a) le Rhône à Jons, b) Le Rhône à Arles. En bleu les données issues de centrifugation, en rouge les données du piège à particules, en pointillés le cumul moyen annuel. 
considérée. En novembre par exemple, les flux de $\mathrm{Hg}$ varient de $11 \%$ entre les 6 méthodes de reconstitution de la chronique de mercure issue de centrifugation, alors qu'ils sont identiques pour la chronique de mercure issue du piège à particules. Ces variations s'expliquent, d'une part, par un taux de lacunes important dans la chronique de $\mathrm{Hg}$ issue de centrifugation $(85 \%$ de lacunes liées à une panne de la centrifugeuse alors que la chronique issue du piège est complète sur ce mois) et, d'autre part, par des différences de concentration en Hg sur les MES prélevées par centrifugeuse et par piège.

A Arles, la différence entre les flux mensuels de $\mathrm{Hg}$ la plus élevée atteint $7,7 \%$ en août 2013. Etant donné que la chronique de MES est complète, ces variations sont uniquement dues aux différentes méthodes de remplissage des lacunes de la chronique de $\mathrm{Hg}$ qui sont très importantes sur ce mois le plus lacunaire de l'année (deux prélèvements réalisés sur 20 jours d'intervalle, soit seulement $19 \%$ de remplissage).

En conclusion, les tests réalisés sur les stations de Jons et d'Arles montrent que, quelle que soit la méthode d'estimation adoptée, les flux de MES et de Hg particulaire calculés à l'échelle annuelle ne diffèrent pas de plus de $5 \%$. A l'échelle mensuelle, en revanche, la méthode d'estimation choisie peut parfois générer des différences importantes $(51 \%$ pour le flux de MES à Jons et jusqu'à $26 \%$ de différence entre les chroniques de $\mathrm{Hg}$ issues de centrifugation à Jons), qui peuvent s'expliquer par l'importance de la durée des lacunes (en lien avec la méthodologie d'estimation d'une période de lacune, Cf. II.2), l'hydrologie (période de base ou évènement de crue), la variabilité temporelle des concentrations en $\mathrm{Hg}$ et la technique de prélèvements de MES utilisée. Les calculs sur des périodes mensuelles lacunaires sont à éviter.

A la suite de ces tests, les flux de MES instantanés et cumulés seront calculés à partir des chroniques de MES estimées par une relation débit/MES, jugée plus adaptée car elle tient compte des variabilités des concentrations liées à l'hydrologie. Les tests réalisés à Arles montrent que les estimations par la relation débit/MES et par le couplage de la relation débit/MES au modèle Rhône 1D sont similaires. Néanmoins, l'utilisation de données simulées peut, dans certains cas, améliorer l'estimation de la chronique de MES en incluant des évènements hydrologiques majeurs non pris en compte dans la relation débit/MES seule (crue de l'Isère par exemple). Les flux de $\mathrm{Hg}$ particulaire seront calculés préférentiellement à partir des séries temporelles de $\mathrm{Hg}$ issues de piège à particules, reconstituées par une méthode par palier centré sur le point de prélèvement.

\section{DISCUSSION ET PERSPECTIVES}

Les séries temporelles hydrologiques sont essentielles à la stratégie d'observation des flux particulaires développée dans l'OSR. Associées à des mesures en continu de paramètres physico-chimiques, elles permettent de dresser des bilans instantanés et cumulés de concentrations en MES et de contaminants particulaires en transit dans le Rhône. Les calculs de flux sont réalisés automatiquement grâce à l'outil $\mathrm{BDOH}$, à différentes échelles temporelles et de manière homogène entre toutes les stations et tous les partenaires de l'OSR. Cependant, les inévitables lacunes dans les séries mères de débit et de concentrations sont propagées dans les chroniques de flux, rendant impossibles les calculs automatiques de flux cumulés sur les périodes incomplètes dans BDOH. Plusieurs techniques de reconstitution des lacunes (interpolation linéaire, relation débit/MES, teneur moyenne de mercure par gamme de débit) ont été utilisées pour tester la sensibilité de la méthode d'estimation des séries temporelles sur les calculs de flux particulaires instantanés et cumulés. A l'échelle annuelle, les résultats montrent que la méthode de reconstitution des lacunes utilisée a une influence négligeable sur les flux cumulés de MES et de Hg particulaire. A l'échelle mensuelle, ces différences sont importantes lorsque les chroniques mères sont très lacunaires avec de fortes variations entre les mesures encadrant la période de lacune. Les périodes de lacunes sont donc plus problématiques lorsqu'elles apparaissent durant un évènement hydrologique plutôt qu'en régime de base. Le calcul de cumuls de MES sur une période inférieure à un mois est possible si la chronique de flux de MES instantanée est complète. Ces conclusions étant dépendantes des deux sites étudiés (le Rhône à Jons et à Arles), le test sera élargi aux stations des principaux affluents du Rhône (Arve, Saône, Isère, Durance) et à d'autres contaminants ( $\mathrm{PCB}$, carbone organique particulaire). Les résultats permettront de choisir une option d'estimation de chaque paramètre " parent \% pour une station donnée.

A terme, les incertitudes quantitatives sur les flux particulaires seront estimées en considérant la part du flux qui résulte de données estimées par rapport aux données mesurées. Une des perspectives sera d'utiliser BaRatin pour maîtriser l'ensemble des incertitudes liées à la stratégie d'observation des flux. Les incertitudes sur la mesure de turbidité et sur la courbe de calibration turbidité/MES des capteurs seront propagées sur la série temporelle estimée de concentration en MES. Les incertitudes associées aux concentrations en contaminants seront également prises en compte dans les calculs de flux de contaminants particulaires.

L'originalité de la stratégie d'observation des flux développée dans l'OSR réside dans le suivi en continu des concentrations en MES et dans l'échantillonnage régulier de MES pour l'analyse de contaminants particulaires, afin de prendre en compte les variations temporelles et hydrologiques des cours d'eau. La présentation de la stratégie d'estimation des flux s'est focalisée ici sur les deux stations principales de l'OSR (Le Rhône à Jons et Arles). Les stations permanentes et temporaires sur les affluents du Rhône disposent d'un suivi des flux allégé par rapport à ces deux stations, qui pourrait être étendu à un réseau opérationnel au niveau national. Ces stations hydrométriques sont enrichies d'un turbidimètre, simple d'utilisation et d'entretien, couplé à un préleveur automatique d'eau pour construire la courbe de conversion turbidité/MES du capteur avec le logiciel BaRatin. La série temporelle générée est validée avec le logiciel $\mathrm{DP}+$ de la même manière qu'une chronique de hauteur d'eau pour la mesure de débit. Ces méthodes de transformation et de validation de chroniques à l'aide d'outils tels que $\mathrm{DP}+$ et BaRatin sont déjà utilisées par le réseau hydrométrique national. En parallèle, un piège à particules est installé pour collecter des échantillons de MES une fois par mois, avec quelques centrifugations de contrôle (1 à 4 par an). Les échantillons sont stockés de manière appropriée dans une échantillothèque. Les analyses des substances d'intérêt sont ciblées sur les échantillons les plus pertinents, avec une intercomparaison sur quelques échantillons tests.

\section{REMERCIEMENTS}

Cette étude a été menée dans le cadre du projet Observatoire des Sédiments du Rhône (ZABR), financé par 
le Plan Rhône, FEDER, CNR, EDF, l'Agence de l'eau Rhône Méditerranée Corse, la Région PACA, la Région Rhône-Alpes et la Région Languedoc-Roussillon. Les auteurs remercient tous les partenaires d'Irstea, du Cerege, de l'IRSN et du MIO pour leur contribution, les prélèvements, les analyses chimiques et le développement de l'outil BDOH.

\section{RÉFÉRENCES}

AFNOR (2016) - NF EN ISO 7027-1. Qualité de l'eau Détermination de la turbidité - Partie 1: Méthodes quantitatives, $21 \mathrm{p}$.

AfNOR (2005) - NF EN 872. Qualité de l'eau - Dosage des matières en suspension - Méthode par filtration sur filtre en fibres de verre, $16 \mathrm{p}$.

Branger F., Thollet F., Crochemore M., Poisbeau M., Raidelet N., Farissier P., Lagouy M., Dramais G., Le Coz J., Guerin A., Tallec G., Peschard J., Mathys N., Klotz S., Tolsa M. (2014) - Le projet base de données pour les observatoires en hydrologie : un outil pour la bancarisation, la gestion et la mise à disposition des données issues des observatoires hydrologiques de long terme à Irstea. La Houille Blanche, 1 : 33-38.

Dugué V., Walter C., Andries E., Launay M., Le Coz J., Camenen B., FAURE J.-B. (2015) - Accounting for hydropower schemes' operation rules in the $1 \mathrm{D}$ hydrodynamic modeling of the Rhône River from Lake Geneva to the Mediterranean Sea. E-proceedings of the 36th IAHR World Congress, 28 June 3 July, 2015, The Hague, the Netherlands.
Ferrand E., Eyrolle F., Radakovitch O., Provansal M. Dufour S., Vella C., Raccasi G., Gurriaran R. (2012) Historical levels of heavy metals and artificial radionuclides reconstructed from overbank sediment records in lower Rhône River (South-East France). Geochim. Cosmochim. Acta, 82 : 163-182.

Horowitz A. (2008) — Determining annual suspended sediment and sediment-associated trace element and nutrient fuxes. Science of the Total Environment, 400 : 315-343.

LAUnAY M. (2014) - Flux de matières en suspension, de mercure et de PCB particulaires dans le Rhône, du Léman à la Méditerranée, thèse de doctorat, Université Claude Bernard Lyon 1, France, $478 \mathrm{p}$.

Le Coz J., Renard B., Bonnifait L., Branger F., Le Boursicaud R. (2014) - Combining hydraulic knowledge and uncertain gaugings in the estimation of hydrometric rating curves: A Bayesian approach. Journal of Hydrology, 509 : 573-587.

Navratil O., Esteves M., Legout C., Gratiot N., Nemery J., Willmor S., Grangeon T. (2011) - Global uncertainty analysis of suspended sediment monitoring using turbidimeter in a small mountainous river catchment. Journal of Hydrology, 398 : 246-259.

Schulze T., Ricking M., Schroter-Kermani C., Koerner A., Denner H.-D., Weinfurtner K., Winkler A., Pekdeger A. (2007) - The German Environmental Specimen Bank Sampling, processing, and archiving sediment and suspended particulate matter. Journal of Soils and Sediments, 7 : 361-367.

Thollet F., Le Bescond C., Le Coz J., Coquery M., Panay J., Lagouy M., Dramais G. (2015) - Observatoire des Sédiments du Rhône, Irstea. https://doi.org/10.17180/OBS.OSR. 